\title{
Direct Visualization of Mechanical Beats by Means of an Oscillating Smartphone
}

Marcos H. Giménez, Isabel Salinas, Juan A. Monsoriu, and Juan C. Castro-Palacio

Citation: The Physics Teacher 55, 424 (2017); doi: 10.1119/1.5003745

View online: http://dx.doi.org/10.1119/1.5003745

View Table of Contents: http://aapt.scitation.org/toc/pte/55/7

Published by the American Association of Physics Teachers

\section{Make the Switch: Modular Circuits}

Untangle your circuits $\underset{\text { ftrom }}{\text { fring }} \$ 159$
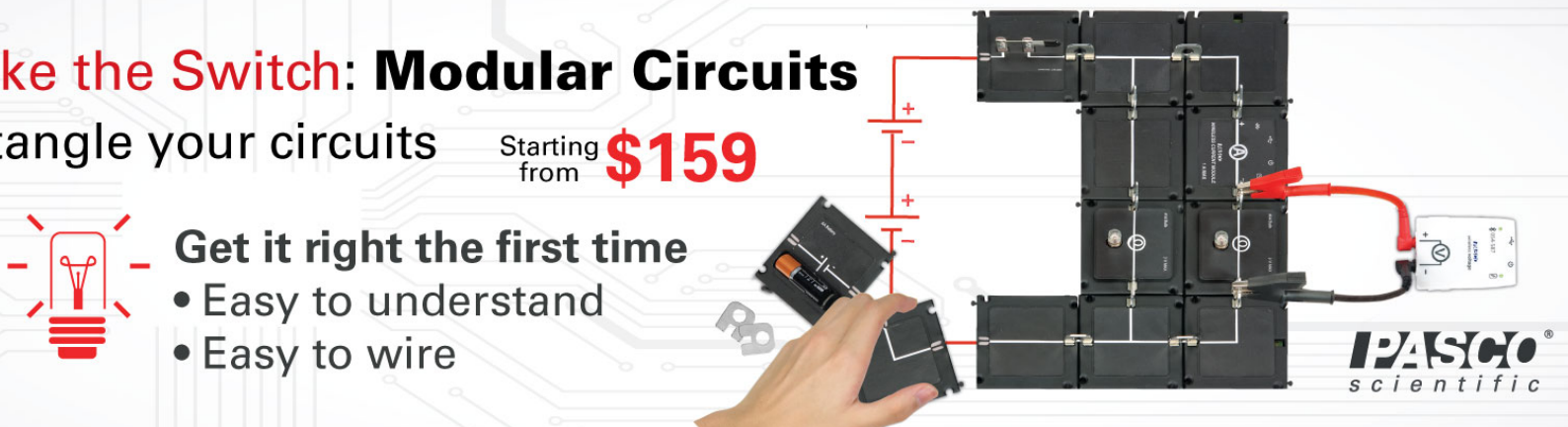


\section{Direct Visualization of Mechanical Beats by Means of an Oscillating Smartphone}

Marcos H. Giménez, Isabel Salinas, and Juan A. Monsoriu, Universitat Politècnica de València, València, Spain Juan C. Castro-Palacio, Imperial College, London, UK

$\mathrm{T}$ he resonance phenomenon is widely known in physics courses. ${ }^{1}$ Qualitatively speaking, resonance takes place in a driven oscillating system whenever the frequency approaches the natural frequency, resulting in maximal oscillatory amplitude. Very closely related to resonance is the phenomenon of mechanical beating, which occurs when the driving and natural frequencies of the system are slightly different. The frequency of the beat is just the difference of the natural and driving frequencies. Beats are very familiar in acoustic systems. There are several works in this journal on visualizing the beats in acoustic systems. ${ }^{2-4}$ For instance, the microphone and the speaker of two mobile devices were used in previous work ${ }^{2}$ to analyze the acoustic beats produced by two signals of close frequencies. The formation of beats can also be visualized in mechanical systems, such as a mass-spring system ${ }^{5}$ or a doubledriven string. ${ }^{6}$ Here, the mechanical beats in a smartphonespring system are directly visualized in a simple way. The frequency of the beats is measured by means of the acceleration sensor of a smartphone, which hangs from a spring attached to a mechanical driver. This laboratory experiment is suitable for both high school and first-year university physics courses.

\section{Equations governing the beats}

From the experimental setup in Fig. 1, Newton's second law can be stated as follows:

$$
F_{0} \sin (\Omega t+\Phi)-k y=m a,
$$

where $F_{0} \sin (\Omega t+\Phi)$ is the driving force acting on the system, $F_{0}$ is its amplitude, $\Omega$ its frequency, and $\Phi$ the initial phase. The term $-k y$ represents the elastic force exerted by the spring on the smartphone. On the right-hand side of the equation are the mass of the smartphone $m$ and its acceleration $a$. The natural frequency of the system is

$$
\omega_{0}=\sqrt{\frac{k}{m}} \text {. }
$$

The solution of the above equation for a free oscillating system, that is, without driving forces $\left(F_{0}=0\right)$, can be expressed as

$$
y_{1}(t)=A \sin \left(\omega_{0} t+\phi\right) .
$$

Equation (2) is the solution of the homogeneous equation governing the oscillations [Eq. (1)]. Moreover, we can define a particular solution of Eq. (1) as ${ }^{7}$

$$
y_{2}(t)=D \sin (\Omega t+\delta)
$$

where the amplitude is

$$
D=F_{0} /\left(m\left|\Omega^{2}-\omega_{0}^{2}\right|\right) .
$$

Finally, the general solution of Eq. (1) is then given by the sum of both solutions given in Eqs. (2) and (3),

$$
y(t)=A \sin \left(\omega_{0} t+\phi\right)+D \sin (\Omega t+\delta) .
$$

The beats appear in the system of Fig. 1 when the frequencies $\Omega$ and $\omega_{0}$ in Eq. (5) are only slightly different. In our experimental setup, the oscillation data are captured with the accelerometer of the smartphones. In this respect, the expression for the acceleration of the system should be defined from Eq. (5) as

$$
a(t)=\frac{d^{2} y}{d t^{2}}=B \sin \left(\omega_{0} t+\phi\right)+E \sin (\Omega t+\delta),
$$

where $B=-\omega_{0}^{2} A$ and $E=-\Omega^{2} D$. Using some trigonometric identities, Eq. (6) can be rewritten as

$$
\begin{aligned}
& a(t)=C \sin \left(\frac{\Omega+\omega_{0}}{2} t+\phi_{\mathrm{s}}\right), \\
& \text { with } C=\sqrt{B^{2}+E^{2}+2 B E \cos \left(\left|\Omega-\omega_{0}\right| t+\phi_{\mathrm{c}}\right)} .
\end{aligned}
$$

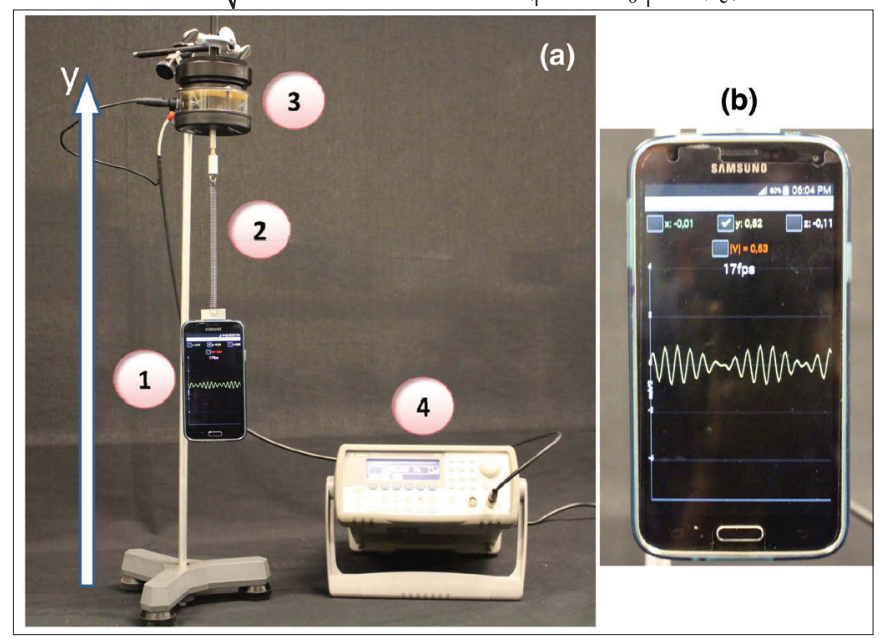

Fig. 1. The experimental setup consisting of the smartphone (1), the spring (2), mechanical driver (3), and the AC generator (4) is included in panel (a). The smartphone has been attached to the spring by means of a small metal plaque and double-sided adhesive tape. A smartphone showing beats on the screen is included on the right-hand side, in panel (b). The $y$-axis is used as a reference axis. 
The phases $\phi_{\mathrm{s}}$ and $\phi_{\mathrm{c}}$ can be derived from the different parameters involved in the system, but this is not relevant for the visualization of the beats in this work. It can be noticed that the frequency in Eq. (7) is the average frequency, $\left(\Omega+\omega_{0}\right) / 2$. On the other hand, the amplitude $C$ is modulated by an envelope curve of frequency $\left|\Omega-\omega_{0}\right|$.

Table I. Driving frequency $(f)$, frequency difference $\left(\left|f-f_{0}\right|\right)$ calculated from the value set at the AC generator minus the natural frequency obtained from the fit [Fig. 2(a)] when the mechanical driver is off, and frequency of the beats $\left(f_{\text {beat }}\right)$ measured on Fig. 2(b). The percentage deviations $(D)$ are included in the last column.

\begin{tabular}{|l|c|c|c|c|}
\hline & $\begin{array}{c}\text { Driving } \\
\text { frequency } \\
f(\mathrm{~Hz})\end{array}$ & $\begin{array}{c}\text { Theoretical } \\
\text { beat } \\
\text { frequency } \\
\left|f-f_{0}\right|(\mathrm{Hz})\end{array}$ & $\begin{array}{c}\text { Experimental } \\
\text { beat } \\
\text { frequency } \\
f_{\text {beat }}(\mathrm{Hz})\end{array}$ & $\begin{array}{c}D \\
(\%)\end{array}$ \\
\hline Exp. I & 2.769 & 0.059 & 0.060 & 1.7 \\
\hline Exp. II & 2.952 & 0.242 & 0.239 & 1.3 \\
\hline
\end{tabular}

\section{Description of the experiments and analysis}

First of all, the mechanical driver is turned off and the natural frequency of the system is determined. The nearly free oscillations captured by the acceleration sensor of the smartphone are shown in Fig. 2(a). The sensor data are collected with the Android application Accelerometer Monitor, which can be downloaded from the Google Play website. ${ }^{8}$ A similar application called Vibsensor is available for iOS. ${ }^{9}$ The value of the natural period, $T_{0}$, directly measured from the plot is $T_{0}=$ $0.369 \mathrm{~s}$, so the natural frequency without the driving force is $f_{0}=1 / T_{0}=2.710 \mathrm{~Hz}$. In the next experiments, the mechanical driver is turned on. The driving frequency is set to $f=$ $2.769 \mathrm{~Hz}$ (experiment I) and $f=2.952 \mathrm{~Hz}$ (experiment II), which corresponds to the difference between the driving and the natural frequencies, $\left|f-f_{0}\right|=\left|\Omega-\omega_{0}\right| /(2 \pi)$, of $0.059 \mathrm{~Hz}$ and $0.242 \mathrm{~Hz}$, respectively. The corresponding data registered with the acceleration sensor for these frequencies are shown in Fig. 2(b). The oscillation beats are clearly seen.

From direct measurements on Fig. 2(b), the values of the beat frequencies $\left(f_{\text {beat }}\right)$ can be measured. Results are included in Table I along with the frequency difference $\left(\left|f-f_{0}\right|\right)$ calculated from the value set at the AC generator minus the natural frequency obtained from the fit [Fig. 2(a)] when the mechanical driver is off. A good agreement is observed-that is to say, the percentage deviations in the last column are lower than $2 \%$ for both cases.

This laboratory experiment is being implemented with success in the first physics course for the Industrial Design Engineering degree at the Universitat Politècnica de València, Spain. We carried out a survey that indicated over $95 \%$ of the students possess a smartphone. The use of these devices, which are very familiar to students, contributes greatly to motivate introductory and first-year university students to physics concepts.
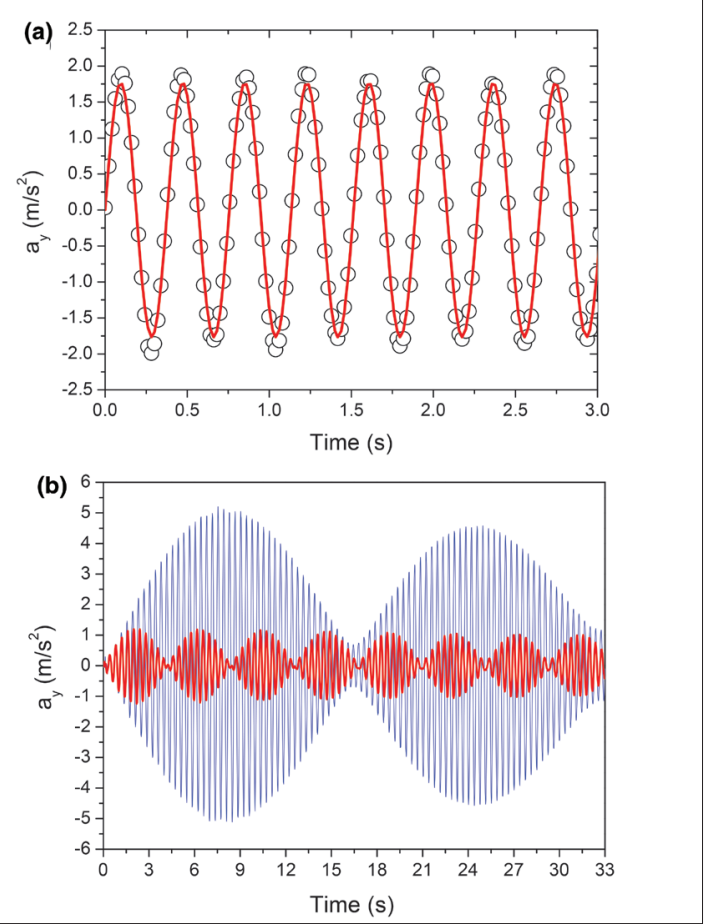

Fig. 2. Free oscillations of the smartphone as recorded by the acceleration sensor (a) and driven oscillations for $\mid \boldsymbol{f}$ $f_{0}=0.059 \mathrm{~Hz}$ (blue line) and $0.242 \mathrm{~Hz}$ (red line) (b).

\section{Acknowledgments}

The authors would like to thank the Institute of Educational Sciences of the Universitat Politècnica de València (Spain) for the support of the Teaching Innovation Groups MoMa and e-MACAFI and for the financial support through the Project PIME 2015 B18.

\section{References}

1. R. Resnick, D. Halliday and K. Krane, Physics, 4th ed. (CECSA, Mexico, 2001).

2. J. Kuhn, P. Vogt, and M. Hirth, "Analyzing the acoustic beat with mobile devices," Phys. Teach. 52, 248-249 (April 2014).

3. A. Ganci and S. Ganci, "The simplest demonstration on acoustic beats," Phys. Teach. 53, 32-33 (Jan. 2015).

4. D. Keeports, "Watching harmonics beat," Phys. Teach. 53, 244 (April 2015).

5. B. Andersson and M. Dykoski, "Demonstrating beats with springs and cart," Phys. Teach. 43, 490-491 (Nov. 2005).

6. C. Carmichael and S. Smith, "Demonstration of beats with a double-driven string," Phys. Teach. 42, 462 (Nov. 2004).

7. C. A. Gaffney and D. Kagan, "Beats in an oscillator near resonance," Phys. Teach. 40, 405-407 (Oct. 2002).

8. Accelerometer Monitor, Google Play, https://play.google.com/ store/apps .

9. Vibesensor, iTunes, https:/itunes.apple.com/us/app/vibsensor-accelerometer-recorder/id $932854520 ? \mathrm{mt}=8$.

Universitat Politècnica de València, València, Spain jmonsori@fis.upv.es 